\title{
Mean Motions and Longitudes in Indian Astronomy
}

\author{
Dennis W. Duke, Florida State University
}

The astronomy we find in texts from ancient India is similar to that we know from ancient Greco-Roman sources, so much so that the prevailing view is that astronomy in India was in large part adapted from Greco-Roman sources transmitted to India. ${ }^{1}$ However, there are sometimes differences in the details of how fundamental ideas are implemented. One such area is the technique for dealing with mean motions and longitudes. The Greek methods explained by Ptolemy are essentially identical to what we use today: one specifies a mean longitude $\bar{\lambda}_{0}$ for some specific day and time - the epoch $t_{0}$ - and uses known mean motions $\omega$ to compute the mean longitude $\bar{\lambda}$ at any other time from the linear relation

$$
\bar{\lambda}=\bar{\lambda}_{0}+\omega\left(t-t_{0}\right) .
$$

Rather than specify a mean longitude $\bar{\lambda}_{0}$ for some epoch $t_{0}$, the methods used in Indian texts from the $5^{\text {th }}$ century and later instead assume the mean or true longitudes of all planets are zero at both the beginning and end of some very long time period of millions or billions of years, and specify the number of orbital rotations of the planets during those intervals, so that mean longitudes for any date may be computed using

$$
\bar{\lambda}=\frac{R}{Y}\left(t-t_{0}\right)
$$

where $R$ is the number of revolutions in some number of years $Y$, and $t-t_{0}$ is the elapsed time in years since some epoch time at which all the longitudes were zero. Such 'great year' schemes may have been used by Greco-Roman astronomers, ${ }^{2}$ but if so we have nothing surviving that explains in detail how such methods worked, or even if they were seriously used. Since the Indian texts give only computational algorithms, and those often cryptically, we never find any explanation of the underlying derivations of the techniques used in those texts. Hence it is of interest to understand is as much detail as possible the methods used to construct the revolution numbers $R$.

In Greek astronomy as exemplified in the Almagest, the units of $\omega$ are generally revolutions per year or degrees per day, and the revolutions can be either sidereal (returns to a specific fixed star) or tropical (returns to a specific cardinal point of the Sun's orbit). The mean motions $\omega$ are determined by dividing the total degrees traveled over many years by the number of days in the time interval or by specifying period relations giving the number of revolutions in longitude and anomaly in, typically, a few decades of years. In the case of the planets, small fractional corrections are also specified. ${ }^{3}$ These period relations are all seen first in Babylonian records and were certainly determined using empirical observations recorded over several centuries. ${ }^{4}$ The positions in mean longitude and anomaly are deduced from involved and complicated comparisons of observed true 
longitudes and the assumed kinematical models. These comparisons give not only the mean positions but also the structural parameters of the models: the longitude of the apogee, the eccentricity of the deferent, and the radius of the epicycle. ${ }^{5}$

For all the planets there are relations between the mean motions in longitude $\omega_{\mathrm{p}}$ and anomaly $\omega_{\mathrm{a}}$ and the mean motion $\omega_{\mathrm{S}}$ of the Sun. In the Almagest the mean position on the epicycle is reckoned with respect to the apogee of the epicycle, which is the point on the epicycle on the extension of the line from the center of uniform rotation to the center of the epicycle, and of course that line is rotating with constant speed. For the outer planets Saturn, Jupiter, and Mars - those that can achieve any elongation from the Sun these mean motions satisfy the relation

$$
\omega_{S}=\omega_{p}+\omega_{a}
$$

For the inner planets Venus and Mercury - those that achieve only limited elongations from the Sun - the conventional mean motion of the planet is exactly the mean motion of the Sun, so at some point it apparently became useful to specify the mean motion according to

$$
\omega_{p}=\omega_{S}+\omega_{a}
$$

so that the mean position on the epicycle is now reckoned with respect to a fixed direction in space, typically either a fixed star or a cardinal point. In modern terms, we of course recognize this as the heliocentric motion of the inner planets, and so in fact under this definition $\omega_{\mathrm{p}}$ is the heliocentric mean motion for both the inner and outer planets.

One consequence of these relations is that an author need specify only $\omega_{p}$ for each planet, and $\omega_{a}$ can be immediately derived. In the Almagest, perhaps for convenience, Ptolemy in fact specifies and tabulates both the mean motion in longitude and anomaly for all the planets, while in the Planetary Hypotheses he is more economical, specifying only the single planetary mean motions $\omega_{\mathrm{p}}$ as defined above for each planet. ${ }^{6}$ All ancient Indian texts on astronomy follow exactly the same scheme of specifying just one mean motion per planet, the $\omega_{\mathrm{p}}$ mentioned above, although, just as in the Almagest, it is explicitly stated that for the outer planets the rotations of the epicycle equal the rotations of the Sun, and for the inner planets the mean motion of the planet equals the mean motion of the Sun. ${ }^{7}$

\section{The Literary Background}

The Indian astronomy texts not only mention very long time intervals, but also give some information on the structure of those intervals. In the Paitamahasiddhanta (hereinafter Paita) and the Brahmasphutasiddhanta (hereinafter BSS), ${ }^{8}$ the fundamental long time interval is a kalpa of $Y=4,320,000,000$ years and the numbers of revolutions, which are the same in both documents, range from $R=146,567,298$ for Saturn to $R=57,753,300,000$ for the Moon. In this system the kalpa is constructed from 
mahayugas of 4,320,000 years according to a rather elaborate scheme: a kalpa consists of 14 manvantaras, and each manvantara consists of 71 mahayugas, or 306,720,000 years. Each manvantara is preceded and followed by one of 15 sandhis or twilight periods consisting of $4 / 10^{\text {th }}$ of a mahayuga, or $1,728,000$ years, and so a kalpa consists altogether of exactly 1,000 mahayugas. Each mahayuga is itself divided into four parts with lengths in the ratios 4:3:2:1, hence a krtayuga of 1,728,000 years (so that a sandhi is a krtayuga), a tretayuga of 1,296,000 years, a dvaparayuga of 864,000 years, and a kaliyuga of 432,000 years. Finally, to connect all this to a 'modern' date, it is said that what is effectively sunrise on -3101 Feb 18 occurred at the beginning of the kaliyuga of the $28^{\text {th }}$ mahayuga of the seventh manvantara, hence after the lapse of six manvantaras and seven sandhis and 27.9 mahayugas, all of which together makes 4567 periods of 432,000 years, or 1,972,944,000 years in total, which is 0.4567 of a kalpa. As we shall see below, the number 4567 is intimately involved in the construction of the revolution numbers. The version of the Paita that survives does not give the connection to a modern date, but it is quite corrupted and from the great overlap with the $B S S$ we can be sure that the Paita, or something closely related to it, was Brahmagupta's source, and that the time details we find described by Brahmagupta were originally in the Paita or the closely related source.

In the Aryabhatiya Aryabhata uses a very similar scheme. He keeps the same mahayuga of 4,320,000 years and gives the revolutions of each planet in a mahayuga, with numbers ranging from 146,564 for Saturn to 57,753,336 for the Moon. However, he then declares that a kalpa is 14 manvantaras and each manvantara is 72 mahayugas, so for him a kalpa is 1,008 mahayugas or 4,354,560,000 years. He further divides the mahayuga into four equal kaliyugas, each of 1,080,000 years. Finally, in his sunrise scheme he says that the same date Brahmagupta used, sunrise on -3101 Feb 18, occurs at the beginning of the final kaliyuga of the $28^{\text {th }}$ mahayuga of the seventh manvantara, hence after the lapse of six manvantaras and 27.75 mahayugas or 1,986,120,000 years, which is about 0.4561 of his kalpa, and which is about as close to Brahmagupta's 0.4567 as Aryabhata could get, given his system. However, as we shall see below, the number 4561 plays no role whatsoever in Aryabhata's construction of the revolution numbers.

The parallels in all these astronomy texts - the final kaliyuga of the $28^{\text {th }}$ mahayuga of the $7^{\text {th }}$ manu of the current kalpa - are obviously striking, and in fact mirror well-established tradition in older Indian literary texts. ${ }^{9}$ The Hindu epic poem Mahabharata, probably compiled in the second half of the first millennium B.C., describes the general scheme of yugas and kalpas in a section that is generally thought to be a late interpolation. ${ }^{10}$ The entire scheme for constructing the kalpa and locating the current kaliyuga within it is described in the Vishnu Purana, ${ }^{11}$ probably compiled sometime between the $1^{\text {st }}$ century B.C. and the $4^{\text {th }}$ century A.D., and everything except the position in the $28^{\text {th }}$ mahayuga is described in the Law Code of Manu, ${ }^{12}$ probably compiled in the first or second century A.D. What is missing from all the literary texts is any information tying the events in those texts, most importantly the date of the battle of Bharata, which occurred at the beginning of the current kaliyuga, with any modern date. However, as we shall see below, the 3600 year interval between the beginning of the current kaliyuga and 499 Mar 21 is given directly or indirectly in the Paita, the BSS, the Aryabhatiya, and in early 
commentaries, so it seems this interval may have been contributed by some astronomer, but we have no way of knowing who it was or when he did it.

\section{Aryabhatiya Revolution Numbers $R$}

Our task now is to understand the construction of the revolution numbers in both systems. The short tradition, exemplified in Aryabhata's sunrise and midnight systems, is to give $\omega$, the mean motion in sidereal longitude, as the ratio of two large integers,

$$
\omega=\frac{R}{Y}
$$

where $R$ is the integral number of sidereal revolutions in each mahayuga of length $Y$ years. Aryabhata stipulates that all the mean longitudes are zero at the beginning and end of each mahayuga, and in addition, at the beginning of the $4^{\text {th }}$ of the four equal kaliyugas that make up each mahayuga. This condition requires that $R$ must be divisible by four. The beginning of the fourth kaliyuga is at the date

$$
t_{0}=-3101 \text { Feb } 18
$$

either midnight or sunrise, at approximately $76^{\circ}$ east longitude. Thus the mean longitude in revolutions after an interval $\Delta t=t-t_{0}$ will be of the form

$$
\bar{\lambda}=\omega\left(t-t_{0}\right)=R^{\prime}+r^{\prime}
$$

where $R^{\prime}$ is the integral number of revolutions accomplished and $r^{\prime}$ is the fraction of a revolution accomplished over and above $R^{\prime}$, and so $\bar{\lambda}$ in degrees is just $360^{\circ} \times r^{\prime}$. As explained above, for Mercury and Venus the mean longitude is just the mean longitude of the Sun, so instead $\bar{\lambda}$ refers to the longitude of the epicycle radius, referred to a fixed direction in space (i.e. not the epicycle apogee, as we see in the Almagest).

Now Aryabhata asserts that in the sunrise system 4,320,000 years contains 1,577,917,500 days, while the same number of years in the midnight system contains 1,577,917,800 days. Thus the sidereal year length in the sunrise system is $365 ; 15,31,15$ days, while in the midnight system it is 365;15,31,30 days, and so in both cases adding 3,600 of the respective years brings us to the same modern date: 499 Mar 21 at noon. Both year lengths are longer than the real sidereal year of 365;15,22,54 days.

We can therefore determine the mean longitudes of the planets in Aryabhata's systems on

499 Mar 21 at noon by computing $\bar{\lambda}=3,600 \times \omega$. Since 3,600 is sexagesimally expressed as 1,0,0 the calculation is most transparent if we also express $\omega$ in a sexagesimal base. Since in both systems $R$ must be an integer divisible by 4, $\omega$ must be of the form 


$$
\omega=n ; \alpha, \beta, \gamma, \delta
$$

where $\delta$ must be either $0,12,24,36$, or 48 . Then we can find $\bar{\lambda}$ by a simple double leftshift:

$$
\bar{\lambda}=\omega\left(t-t_{0}\right)=1,0,0 \times \omega=n, \alpha, \beta ; \gamma, \delta
$$

so $R^{\prime}=n, \alpha, \beta$ revolutions and $r^{\prime}=0 ; \gamma, \delta$ revolutions, or, in degrees,

$$
\bar{\lambda}=360^{\circ} \times 0 ; \gamma, \delta=6^{\circ} \times \gamma ; \delta .
$$

Since $\delta$ is a multiple of $12, \bar{\lambda}$ will be an integral multiple of $1 ; 12^{\circ}$, and if $R$ changes by \pm 4 revolutions, then $\bar{\lambda}$ changes correspondingly by $\pm 1 ; 12^{\circ}$. Note also that if Aryabhata had kept the traditional 4:3:2:1 ratios his kaliyuga would have been 432,000 years instead of 1,080,000 years, and hence his rotation numbers must have been divisible by 10 and his granularity would be $3^{\circ}$, which he might have regarded as too large for useful work. There is some evidence that such a scheme was at some point related to the midnight system and was influential in the creation of the zij al-Arkand, which is one of the earliest transmissions of Indian astronomy into Islam. ${ }^{13}$

Using the $R$ values from Aryabhata's sunrise system, one finds for Saturn

$$
\omega=\frac{146,564}{4,320,000}=0 ; 2,2,8,12
$$

so the mean longitude is simply $6^{\circ} \times 8 ; 12=49 ; 12^{\circ}$ and the leading two digits $(2,2)$ are what one expects from the well-known period relation $\frac{59-57}{59}=0 ; 2,2,2 \ldots$.

For Jupiter one finds

$$
\omega=\frac{364,224}{4,320,000}=0 ; 5,3,31,12
$$

so the mean longitude is $6^{\circ} \times 31 ; 12=187 ; 12^{\circ}$, and the leading two digits 5,3 are consistent with the well-known period relation $\frac{83-76}{83}=0 ; 5,3,36 \ldots$ For the midnight system $R$ for Jupiter is 364,220 , and so the corresponding mean longitude is decreased to $186^{\circ}$.

For Mars, 


$$
\omega=\frac{2,296,824}{4,320,000}=0 ; 31,54,1,12
$$

so the mean longitude is $6 \times 1 ; 12=7 ; 12^{\circ}$. The leading two digits 31,54 are consistent with the common period relations for Mars:

$$
\frac{79-37}{79}=0 ; 31,53,55 \ldots, \frac{284-133}{284}=0 ; 31,54,5 \ldots
$$

For Venus,

$$
\omega=\frac{7,022,388}{4,320,000}=1 ; 37,31,59,24
$$

so the mean longitude is $6^{\circ} \times 59 ; 24=356 ; 24^{\circ}$. Using Venus' familiar short period relation gives $\frac{5+8}{8}=1 ; 37,30$ (exactly), while a longer one gives $\frac{152+243}{243}=1 ; 37,31,51$ suggesting that the longer relation for Venus was used to construct $R$.

For Mercury,

$$
\omega=\frac{17,937,020}{4,320,000}=4 ; 9,7,31,0
$$

so the mean longitude is $6^{\circ} \times 31 ; 0=186^{\circ}$, and the leading digits $4 ; 9,7$ are consistent with the period relation $\frac{46+145}{46}=4 ; 9,7,49$. For the midnight system $R$ for Mercury is $17,937,000$, and so the corresponding mean longitude is decreased to $180^{\circ}$.

For the Sun, there is one revolution per year, and so the longitude of the Sun is zero on both -3101 Feb 18 at sunrise and 499 Mar 21 at noon, the first by construction, the second as a consequence of being exactly 3,600 years later.

For the Moon the short systems specify 57,753,336 rotations in longitude, 488,219 rotations of the lunar apogee, and 232,226 rotations of the lunar node. The node rotates in the clockwise direction, so the rotations will be counted as negative. While the longitudes of the Sun and Moon are, like the five planets, zero on -3101 Feb 18, the Moon's apogee is at $90^{\circ}$ and the ascending node is at $180^{\circ}$. Thus the rotations in longitude are divisible by four as usual, while the rotations of the apogee are three plus some multiple of four, and the rotations of the node are two plus some multiple of four. 
For the Moon,

$$
\omega=\frac{57,753,336}{4,320,000}=13 ; 22,7,46,48
$$

so the mean longitude is $6^{\circ} \times 46 ; 48=280 ; 48^{\circ}$, and the leading digits $13 ; 22,7$ are consistent with the period relation $\frac{235+19}{19}=13 ; 22,6$.

For the Moon's apogee, the revolutions are three plus some multiple of four in order that the longitude of the apogee be $90^{\circ}$ on -3101 Feb 18 . Hence

$$
\omega=\frac{488,219}{4,320,000}=0 ; 6,46,50,57
$$

and so the mean longitude of the apogee is $90^{\circ}+6^{\circ} \times 50 ; 57=35 ; 42^{\circ}$, and the leading digits 0;6,46 are consistent with the relation $\frac{254}{19}-\frac{235}{19} \times \frac{269}{251}=0 ; 6,46$ obtained by using the well-known relation 269 anomalistic months equals 251 synodic months.

For the Moon's node, the revolutions are two plus some multiple of four in order that the longitude of the node be $180^{\circ}$ on -3101 Feb 18 . Hence

$$
\omega=\frac{-232,226}{4,320,000}=-0 ; 3,13,31,18
$$

so the mean longitude of the node is $180^{\circ}-6^{\circ} \times 31 ; 18=-7 ; 48^{\circ}$, and the leading digits $0 ; 3,13$ are consistent with the period relation $\frac{254}{19}-\frac{235}{19} \times \frac{5923}{5458}=-0 ; 3,13$ obtained by using the well-known relation 5923 draconitic months equals 5458 synodic months.

These deconstructions of the rotation numbers $R$ for the five planets clearly suggest how the numbers might have been originally constructed: from the period relations one would compute $n ; \alpha, \beta$, from the mean longitudes, rounded to the nearest multiple of $1 ; 12^{\circ}$, one would get $\gamma, \delta$, and thus compute $R$ as

$$
R=4,320,000 \times n ; \alpha, \beta, \gamma, \delta=20,0,0 \times n ; \alpha, \beta, \gamma, \delta
$$

Clearly the revolution numbers $R$ contain exactly two components: (1) knowledge of widely known planetary period relations, and (2) rounded mean longitudes at noon on 499 Mar 21. The perfectly clean separation of these components in the sexagesimal construction certainly suggests a strong Greek influence. Also note that because of the 
required rounding, we know only intervals of width $1 ; 12^{\circ}$ that the author thought the mean longitudes were in on 499 Mar 21, and so we have no information where in those intervals his assumed mean longitudes might lie.

If the author had some estimate of a mean longitude at some date other than 499 Mar 21 he could still easily construct the $R$ values. For example, let us use Saturn as an example. In $Y=4,320,000$ years Saturn must make about

$$
R_{0}=\operatorname{int}\left(\frac{2}{59} Y\right)
$$

revolutions plus some fractional rotation, and similarly in $y=3,600+t$ years it will make about

$$
r_{0}=\operatorname{int}\left(\frac{2}{59} y\right)
$$

revolutions plus some fraction $c$ of an additional revolution. Then on the date $t$ years, positive or negative, from 499 Mar 21 noon, we must have

$$
\frac{R}{Y} y=\frac{R_{0}+4 n}{Y} y \simeq r_{0}+c
$$

for some integer $n$, from which, since $c$ is known (it is just the mean longitude on the date $y$ ), one can easily solve for $n$, and hence know $R=R_{0}+4 n$.

There are two simple consequences of this procedure. First, there is no requirement that the mean longitudes be known on the same date. In fact, they can all be known on different dates, and those can be any date whatsoever. Second, whatever the date and whatever the fraction $c$, at the end of the procedure the only thing we know is the value of $R$. All knowledge of the date $t$, the value of $c$, and even the exact details of the period relation (i.e. the 2/59 used above) have been completely erased except for the obvious fact that whatever they were, they led our author to the text value of $R$. And as we have seen above, the only information in $R$ is the leading digits of a period relation and the interval of width $1 ; 12^{\circ}$ into which the mean longitude falls on exactly 499 Mar 21 noon.

\section{Paitamahasiddhanta and Brahmasphutasiddhanta Revolution Numbers $R$}

The system for constructing the revolution numbers for the long time intervals is similar to the short time interval systems, but differs in details and is generally more complex. The revolution numbers in the Paita and the BSS are identical. The fundamental period is $4,320,000,000$ years, or 1,000 times longer. Not only are the mean longitudes zero at the beginning and end of this period, but so also are the apogees and nodes of all the planets, and so the beginning and end of the kalpa is a true conjunction of all the planets, not just a mean conjunction. As explained earlier, the beginning of the current kaliyuga is said to occur when $0.4567 \times 4,320,000,000=1,972,944,000$ years have passed. The number of rotations in 4,320,000,000 years is adjusted so that (a) the mean longitude of each planet 
lies in a desired interval at some contemporary date, and (b) the mean longitudes are all near, but not exactly equal to, zero at the beginning of the current kaliyuga. The number of days in 4,320,000,000 years is said to be $1,577,916,450,000$, implying a sidereal year length of 365;15,30,22,30, slightly shorter than the year lengths in the short system, and 3,600 of these years brings us to 499 Mar 20 at 3 PM, or 21 hours earlier than Aryabhata's modern date.

The construction of the $R$ values in the long system is perhaps best explained with an example. Let us use Saturn, which according to the texts makes 146,567,298 rotations in $4,320,000,000$ years. We can find the implied mean longitude at the modern date by computing

$$
\frac{146,567,298}{4,320,000,000}(1,972,944,000+3,600)=66,937,407.136
$$

so that

$$
\bar{\lambda}=360^{\circ} \times 0.136=48 ; 58^{\circ},
$$

which is close to the value $49 ; 12^{\circ}$ that results in Aryabhata's systems, and in fact, given the $\pm 0 ; 36^{\circ}$ degree granularity in those systems, it is effectively the same value. For the long system the implied mean longitude of Saturn on $-3101 \mathrm{Feb} 18$ is about $-1 ; 13^{\circ}$, and thus fairly close to the target value (and Aryabhata's enforced value) of $0^{\circ}$. Of course, both the mean and true longitudes of Saturn and indeed all the planets are exactly zero at the beginning and end of each kalpa.

Since Saturn makes about 2 revolutions in longitude in 59 years, we might expect that $R$ will be about $2 / 59 \times 4,320,000,000 \simeq 146,440,678$ revolutions. However, using that number of revolutions, after 1,972,944,000 years Saturn will have made some $66,879,457.6426$ revolutions, and so have a mean longitude of about $360^{\circ} \times 0.6426=213 ; 20^{\circ}$ on $-3101 \mathrm{Feb} 18$, very far from the target value of zero. Furthermore, after an additional 3,600 years Saturn will have made an additional 122.0339 revolutions and so have a mean longitude of about $243.32^{\circ}$, while we know that the mean longitude should be about $49^{\circ}$.

Clearly we must adjust the number of revolutions, and the first thing to do is to add enough revolutions to our initial estimate, 146,440,678, to eliminate the fractional part of the revolutions at $-3101 \mathrm{Feb} 18 .{ }^{14}$ To this end, let $\rho=146,440,678$ and let us imagine adding $p$ revolutions to $\rho$ so that $0.4567(\rho+p)$ is some integer $m$. We already know that $0.4567 \rho$ is an integer $n$ plus a fractional part $c=0.6426$, so altogether we must have

$$
\begin{aligned}
m & =0.4567(\rho+p) \\
& =n+c+0.4567 p
\end{aligned}
$$

or 


$$
\begin{aligned}
q & =m-n \\
& =c+0.4567 p \\
& =0.6426+0.4567 p
\end{aligned}
$$

Multiplying through by 10,000 we see that we must solve the indeterminate equation

$$
10,000 q-4567 p=6426
$$

Now Greek and Indian mathematicians were certainly able to solve indeterminate equations such as

$$
a q-b p=c
$$

where $a, b$, and $c$ are given integers. One method is to expand the rational fraction $a / b$ in a continued fraction series, which is in itself a simple extension of Euclid's algorithm. The resulting series of convergents will of course terminate in $a / b$ itself, and the penultimate convergent, $p / q$, will be the solution of

$$
a q-b p=1
$$

Letting $p_{0}, q_{0}$ be the particular solution with smallest positive values of $p$, $q$, general solutions may be found from

$$
\begin{aligned}
p & =p_{0}+a t \\
q & =q_{0}+b t, \quad t=0, \pm 1, \pm 2, \ldots
\end{aligned}
$$

So having found $p_{0}, q_{0}$, clearly $c p_{0}, c q_{0}$ will be a general solution to $a q-b p=c$ and other solutions will be of the form

$$
\begin{aligned}
& c p_{0}+a t \\
& c q_{0}+b t, \quad t=0, \pm 1, \pm 2, \ldots
\end{aligned}
$$

and the smallest positive solution will be when $-t$ is the integer part of the smaller of $c p_{0} / a$ and $c q_{0} / b$.

Applying this to the equation at hand, we find that the particular solution of $a q-b p=1$ is $\left(p_{0}, q_{0}\right)=(1097,501)$. Then $c p_{0}=7,049,322$ and so the smallest positive solution for $p$ in $a q-b p=c$ is 9,322 so we now know that

$$
\begin{aligned}
R & =\rho+p \\
& =146,440,678+9,322 \\
& =144,450,000
\end{aligned}
$$


is a number of revolutions that will give a mean longitude of zero after 1,972,944,000 years.

Of course these trailing zero digits are obvious in hindsight, and might well have been obvious to whoever invented this scheme. Because 1,972,944,000/4,320,000,000 is exactly a four-digit decimal fraction, namely 0.4567 , if we choose for $R$ any multiple whatsoever of 10,000 , then

$$
R \times \frac{1,972,944,000}{4,320,000,000}
$$

will always be an integer number of rotations, and the mean longitude on -3101 Feb 18 will always be zero.

Our final step is to adjust the trailing digits of $R=146,450,000$ to give a desired mean longitude at our 'modern' date, 3,600 years after $-3101 \mathrm{Feb} 18$. It would be nice if we could adjust only the integers just before the final four zeros, but as it happens, adjusting those digits by one unit changes the mean longitude at our modern date by $\pm 3^{\circ}$, which is rather coarse. In fact, if we set $R=146,400,000+10,000 t, t=0 \ldots 119$, so that $R$ ranges between 146,400,000 and 147,590,000, then the mean longitude at the modern date will assume the 120 values $0^{\circ}, 3^{\circ}, 6^{\circ}, \ldots ., 354^{\circ}, 357^{\circ}$. Now the sexagesimal expansions of $146,400,000$ and 147,590,000 divided by 4,320,000,000 are precisely 0;2,2,0,0 and $0 ; 2,2,59,30$, so we see that exactly as in the analysis of the short systems built on $4,320,000$ years, the first two sexagesimal digits, and only those two (plus the units digit, if it happens to be present), are determined exclusively by the period relation, and all further digits are used simply to encode into $R$ the value of the mean longitude at the modern date. Therefore the separation in function of the sexagesimal digits that we observed in Aryabhata's systems is duplicated exactly in the systems with a factor 1,000 longer time period.

We can encode the mean longitude of Saturn at 3,600 years past $-3101 \mathrm{Feb} 18$ to $48^{\circ}$ by choosing $R$ provisionally as $146,560,000$. To finally adjust the mean longitude at the modern date to some granularity smaller than $3^{\circ}$ we must adjust the final four digits, which are now zero, but this must be done carefully. For example, if we simply add one rotation to get $R=146,560,001$, then the mean longitude at the modern date will be $212 ; 24^{\circ}$ and the mean longitude at -3103 Feb 18 will be $164 ; 25^{\circ}$.

The key clue we need to deal with this extreme sensitivity is already implicit in the analysis given above, where it was shown that the solution of

$$
10,000 q-4567 p=1
$$

is $\left(p_{0}, q_{0}\right)=(1097,501)$, or 


$$
0.4567 \times 1097=501-\frac{1}{10,000} .
$$

This means that if we add the final four digits of a small multiple of 1097 to our provisional $R=146,560,000$ we can keep the mean longitude at -3103 Feb 18 close to zero (we lower it by $1 / 10,000$ rotation, or $0 ; 2,9^{\circ}$, for each added multiple of 1097).

Furthermore, we see that

$$
360^{\circ} \times \frac{3,600}{4,320,000,000} \times 1097=0 ; 19,44^{\circ}
$$

and so the net increase above $48^{\circ}$ in mean longitude after 146,561,097 rotations over $1,972,944,000+3,600$ years is $0 ; 19,44^{\circ}-0 ; 2,9^{\circ}=0 ; 17,35^{\circ}$. Each additional multiple of 1097 rotations that we add increases the net mean longitude by that same amount, and so at the modern date we can get a value between $48^{\circ}$ and $51^{\circ}$ that is acceptably close to our target value for the mean longitude. In the case of Saturn, our author chose 34 multiples of 1097, which is 37,928 , and the final four digits match exactly those in 146,567,298, the number found in the texts. This multiple adds a net $0 ; 58^{\circ}$ to the provisional $48^{\circ}$ for 499 Mar 20, and the mean longitude at -3101 Feb 18 is reduced to 358;47.

We have seen that adding a single multiple of 1097 to any $R$ (or equivalently, subtracting the same multiple of $8903=10,000-1097)$ will change the resulting mean longitude by about $0 ; 18^{\circ}$ (more accurately, $0 ; 17,35^{\circ}$ ) in these schemes, about $14^{\text {th }}$ the $1 ; 12^{\circ}$ granularity in Aryabhata's schemes. Note that if not for the requirement in the shorter system that the revolutions $R$ be divisible by four, the granularities in both systems would be very nearly equal.

The numbers actually used for each planet in the Paita and the BSS are

$$
\begin{array}{lrlrl}
\text { Saturn } & 34 \times 1097 & \text { yielding } & 146,567,298 & \text { and } \bar{\lambda}=48 ; 58^{\circ}\left(49 ; 12^{\circ}\right) \text {, } \\
\text { Jupiter } & 15 \times 1097 & \text { yielding } & 364,226,455 & \text { and } \bar{\lambda}=187 ; 24^{\circ}\left(187 ; 12^{\circ} \text { or } 186^{\circ}\right) \text {, } \\
\text { Mars } & 26 \times 1097 & \text { yielding } & 2,296,828,522 & \text { and } \bar{\lambda}=7 ; 37^{\circ}\left(7 ; 12^{\circ}\right) \text {, } \\
\text { Venus } & 36 \times 1097 \text { yielding } & 7,022,389,492 & \text { and } \bar{\lambda}=355 ; 33^{\circ}\left(356 ; 24^{\circ}\right) \text {, } \\
\text { Mercury } & 72 \times 1097 \text { yielding } & 17,936,998,984 & \text { and } \bar{\lambda}=177 ; 06^{\circ}\left(186^{\circ} \text { or } 180^{\circ}\right) \text {, }
\end{array}
$$

where the crucial final four digits are in italics and the mean longitudes $\bar{\lambda}$ are for 3600 years following -3103 Feb 18 at 6AM, which is 499 Mar 20 at 3 PM. This means that to compare mean longitudes with the short systems we have to add increments to the results in the long system: $0 ; 26^{\circ}$ for Mars, $1 ; 24^{\circ}$ for Venus, and 3;35 ${ }^{\circ}$ for Mercury, the increments for Jupiter and Saturn being negligible. The resulting values then agree reasonably well with the mean longitudes for the sunrise and midnight systems, given in parentheses above, for the same date. 
In the long period system the Moon's apogee makes 488,105,858 revolutions, and its node makes -232,311,168 revolutions. At -3101 Feb 18, the longitude of the apogee is about $125 ; 30^{\circ}$, and the longitude of the node is about $-153 ; 13^{\circ}$. Since the author requires those longitudes to be far from $0^{\circ}$, the final four digits must be related to some large multiple of 1097, and indeed they are: $6514 \times 1097=7145858$ and $5744 \times 1097=6301168 .^{15}$ After another 3,600 years the longitude of the apogee is about $37 ; 15^{\circ}$ and the longitude of the node is about $-6 ; 34^{\circ}$, so both are fairly close to Aryabhata's values $35 ; 42^{\circ}$ and $-7 ; 48^{\circ}$, the adjustments for the 21 hour time difference being small $\left(3^{\prime}-6^{\prime}\right)$. Note that the long and short period values disagree substantially at -3101 : $90^{\circ}$ vs. $125 ; 30^{\circ}$ for the apogee and $-180^{\circ}$ vs. $-153 ; 13^{\circ}$ for the node, so there was a lot of error to recover in the intervening 3600 years.

For all of the revolution numbers in the long period system it is clear that once again they contain exactly two, and only two, components: (1) knowledge of widely known planetary period relations, and (2) rounded mean longitudes at 3 PM on 499 Mar 20. All other information that might have been used to compute the $R$ values has been lost.

\section{The Apogees and Nodes of the Planets}

The apogees and nodes of the planets in the early texts are given in Table 1. For the short period systems Aryabhata keeps the apogees and nodes at constant longitude, but acknowledges that they have moved to their current positions. His meaning becomes clear when we consider the long period systems, which put all the apogees and nodes at the origin at the beginning of the kalpa, and then specify a few hundred rotations for each one to bring them to their modern position. Since they are moving so slowly it hardly matters whether we compute them at the beginning of the current kaliyuga, -3101 Feb 18 , or 3600 years later. For technical reasons that will become clear below, we have chosen the earlier date for Table 1.

\begin{tabular}{|c|c|c|c|c|}
\hline \multicolumn{5}{|c|}{ APOGEES } \\
\hline & rotations & \multicolumn{3}{|c|}{ longitude } \\
\hline & BSS & BSS & midnight & sunrise \\
\hline Mercury & 332 & $224 ; 47$ & 220 & 210 \\
\hline Venus & 653 & $81 ; 02$ & 80 & 90 \\
\hline Sun & 480 & $77 ; 46$ & 80 & 78 \\
\hline Mars & 292 & $128 ; 18$ & 110 & 118 \\
\hline Jupiter & 855 & $172 ; 16$ & 160 & 180 \\
\hline Saturn & 41 & $260 ; 54$ & 240 & 236 \\
\hline \multicolumn{5}{|c|}{ NODES } \\
\hline & rotations & \multicolumn{3}{|c|}{ longitude } \\
\hline & BSS & BSS & midnight & sunrise \\
\hline
\end{tabular}




\begin{tabular}{|l|r|r|r|r|}
\hline Mercury & -521 & $21 ; 20$ & 20 & 20 \\
\hline Venus & -893 & $60 ; 05$ & 60 & 60 \\
\hline Mars & -267 & $22 ; 00$ & 40 & 40 \\
\hline Jupiter & -63 & $82 ; 03$ & 80 & 80 \\
\hline Saturn & -584 & $103 ; 23$ & 100 & 100 \\
\hline
\end{tabular}

The computation of the rotation numbers for the apogees and nodes is closely related to the method used for the longitudes. Let us take Mercury's apogee as an example. Since the computed longitude is $224 ; 47^{\circ}$ in -3101 , and $224 ; 53^{\circ}$ in A.D. 628 , let us suppose that the author was in fact trying to target the round number $225^{\circ}$ (= 0.625 rotations), i.e. he wants a number of rotations $R$ such that

and so he needs to solve

$$
0.4567 R=m+0.625=n-0.375
$$

$$
10,000 n-4567 R=3750 .
$$

Just as discussed above, he would first solve $10,000 n_{0}-4567 R_{0}=1$ and get $R_{0}=1097$. He would then compute $c=1097 \times 3750=4,113,750$ and subtract off enough multiples of 10,000 to get the minimum positive $R=3750$. Apparently although this is a relatively small number of rotations, it was not small enough to satisfy our author, for he adjusted $c$ slightly to $c=1097 \times 3756=4,110,332$, which gives $R=332$. He could just as well have used $c=1097 \times 3647=4,110,459$, which gives $R=459$, but apparently he did not. Clearly the key is to pick a multiple of 1097 that gives a zero in the $4^{\text {th }}$ decimal place of $c$ (moving right to left), which will happen about every $9^{\text {th }}$ multiple. The target longitudes were apparently $225^{\circ}, 81^{\circ}, 78^{\circ}, 128^{\circ}, 172^{\circ}$, and $261^{\circ}$ for the apogees, and $-20^{\circ},-60^{\circ}$, $22^{\circ},-80^{\circ}$, and $-103^{\circ}$ for the nodes. There is a certain amount of post hoc reasoning in this argument, but it is clearly consistent with what we saw above regarding the construction of the $R$ values for the mean longitudes.

\section{Source of the Mean Longitudes}

We have seen that the rotation numbers in the Indian systems contain two pieces of information: the two leading sexagesimal digits are the same as those arising in standard period relations, and the remaining digits encode the mean longitude at a date 3,600 years after the beginning of the current kaliyuga on -3101 Feb 18. Ideally, we would like to understand the source of the Indian values for mean longitude. There are two extreme possibilities: either the mean longitudes were taken from some available astronomical tables, e.g. those in the Almagest or the Handy Tables, or they were derived from observations made in India around A.D. 500. The principal advocate for the case that tables were used was Pingree, ${ }^{16}$ while the principal advocates for observation in India 
were Billard ${ }^{17}$ and van der Waerden. ${ }^{18}$ It is, of course, also possible that some combination of those strategies was used.

The mean longitudes for 499 Mar 21 noon at $76^{\circ} \mathrm{E}$ from modern theory, the Almagest, and the three major Indian models are given in Table 2. The deviations of the Almagest values and the Indian values from modern theory are given in Table 3 . When comparing the Indian values to any system we must remember that the sunrise and midnight values are always multiples of $1 ; 12^{\circ}$ and the BSS values are always multiples of about $0 ; 18^{\circ}$. In addition, to compare the Indian values to either the modern or Almagest values we must first take into account that the modern values are given in a tropical coordinate system relative to an accurate vernal equinox, the Almagest values are also tropical but relative to a vernal equinox that is inaccurate by several degrees in A.D. 499, while the Indian values are probably sidereal but the texts give no direct information about the location of the zero point. It has been speculated by many people that the Indian zero point is the star $\zeta$ Pisces, or possibly some unstarred point nearby it, but as far as I can tell the only rationale is that in A.D. $499 \zeta$ Pisces was very near the ecliptic and its longitude was about $359^{\circ}$. However, with a visual magnitude of 5.2 it is extremely dim and would be very difficult to use as a reference star.

Let us first consider the case for adapting longitudes from tables. Setting aside for now the uncertainty in the various zero points, we note that on 499 Mar 21 noon the true mean Sun was only $0 ; 14^{\circ}$ shy of zero. If someone had estimated a more accurate length of the tropical year than Ptolemy did, or if relatively accurate sidereal tables from some earlier era were available, then it is possible that such a circumstance around noon on 499 Mar 21 could have been widely expected. The Indian mean Sun was by construction at $0^{\circ}$ longitude that noon, and so the true and Indian mean Suns would be aligned to a reasonable accuracy. At this point, the author of any of the Indian systems need only have an estimate of the elongation of the mean longitude of the planet from the mean Sun in any other system, and this elongation would be the mean longitude in the Indian system. For the planets, these elongations are simply the mean positions in anomaly.

These errors in elongation from the Sun are shown in Table 4. We see that for the sunrise system all the errors except those for Mercury are smaller than the $\pm 0 ; 36^{\circ}$ spread, and the midnight system is similar except for an additional error for Jupiter. The $B S S$ and Almagest errors are similar for most of the planets. The Almagest Mercury and Venus errors are due to inaccurate mean motions in anomaly and for Mercury are quite similar to the errors in the midnight and $B S S$ systems. The Mercury error in the sunrise system is also large, but has the opposite sign. Mercury and Jupiter are the two cases where the mean longitudes change between the sunrise and midnight systems. The errors for Mars, Saturn, and the Moon, including its node and apogee, are in very good agreement for the Almagest, sunrise, and midnight systems, and the overall agreement is not as good with BSS.

Overall, this pattern of errors is clearly consistent with a scenario which includes knowledge of a true mean solar longitude near 499 Mar 21 at noon, plus a set of fairly accurate tables for mean anomaly. Such tables would depend on good values for the 
synodic periods of the planets, and the synodic, anomalistic, and draconitic months for the Moon, plus fairly accurate positions in anomaly at some epoch date. These tables would agree fairly well with the Almagest tables for Mars, Saturn, and the Moon, including its node and apogee, but would be somewhat improved for Venus and Jupiter. Mercury was apparently never gotten right by anyone, but the Almagest, midnight and BSS systems seem to have similar errors.

Let us now consider the opposite extreme position, that all the required mean positions in anomaly were derived from contemporary observation in India. Billard's thesis is that by plotting the deviations in mean longitude as a function of time, one could determine the date of the observations by finding when the deviations, or more precisely their standard deviation, were minimized. Such plots are shown in Figs. 1 and 2.

Now generally speaking, mean longitudes are not directly observable. Rather it is necessary to observe true longitudes, and from these, using a model with known structural parameters such as eccentricity, epicycle radius, and longitude of the apogee, one can compute the desired mean longitudes. In the case of the Indian astronomers, there are at least three main problems:

(1) there is no evidence that the astronomers would be able to measure true longitudes with any accuracy. The surviving lists of star coordinates in the Indian texts are very imprecise and so no measurement of a planet relative to a star would be accurate. Similarly, the Indian models of this era lacked the second lunar anomaly, so measures with respect to the Moon would show very large errors.

(2) the structural parameters given in the texts are often not at all accurate, especially for the planets, and so show no evidence for underlying accurate observations.

(3) the kinematic models used by the Indian astronomers are at best approximations to good models such as the equant, and for Mercury, Venus, and Mars, not very good approximations at that. ${ }^{19}$ Further, there is no known systematic way to derive structural parameters using the Indian approximations, i.e. there is nothing comparable to Ptolemy's iteration scheme for the equant.

These problems make determination of an accurate mean longitude from a set of observed true longitudes very difficult. Let us illustrate these difficulties with the case of Jupiter. ${ }^{20}$ In the midnight system we have $R=364,220$ and the mean longitude is $186^{\circ}$. The comparison of the real longitudes and the predictions of the midnight system over the two synodic periods of the interval A.D. $508-510$ is shown in Fig 3. Of course this figure is already greatly misleading since no observer in that time could possibly make enough observations to draw such smooth curves (nor could they even draw such a comparison graph), and even if they could, observation errors, very likely as large as $1^{\circ}$ $2^{\circ}$, would induce substantial scatter not shown, but easily imagined, in our figure. Nevertheless, let us for now suppose that somehow our astronomer would realize that what is needed is to increase the mean longitude. The smallest adjustment possible is to add four revolutions, making $R=364,224$ as in the sunrise system, and increasing the mean longitude by $1 ; 12^{\circ}$. This gives the middle curve on the chart, which is indeed in 
better agreement with reality, although nowhere near as good as it could be if the other parameter values were better adjusted.

However, this is not all our astronomer actually changed in going to the sunrise system. Small adjustments are made to the eccentricity and the epicycle radius and small pulsations of both radii are added, but on the scale of the inherent uncertainties in the entire process, these changes are inconsequential. What is significant is that the apogee is moved from $160^{\circ}$ to $180^{\circ}$. The resulting predictions of the full sunrise system are shown as the top curve in Figure 3, and if our astronomer really did understand how to adjust his parameters to get better agreement with observation, it is certainly not evident in his treatment of Jupiter. This is further illustrated in Fig 4, which shows the corresponding model errors for both systems for Jupiter over an entire 12-year sidereal period. Similar patterns are seen for the other planets in Figs 5 - 8. There is clearly no evidence of accurate observations being turned into excellent agreement with the sunrise or midnight systems.

Beyond all this, however, the analysis given above of the information content in the revolution numbers $R$ shows that Billard's scheme was in fact futile from the very beginning. The revolution numbers contain exactly two, and only two, components: (1) knowledge of widely known planetary period relations, and (2) rounded mean longitudes on 499 Mar 21 or Mar 20. All other information that might have been used to compute the $R$ values, including the date of any possible observations, has been lost.

Although Billard's deviation plots provide no useful information for this analysis, they might still be useful in other circumstances in which specific dates are not deliberately encoded into the mean motions which determine the slopes of the lines in the plots. ${ }^{21}$

\section{Acknowledgements}

I am grateful to Christopher Minkowski and Luis González-Reimann for help in locating the literary references to the kalpa system in the ancient Hindu texts. 


$\begin{array}{lccccc}\begin{array}{l}\text { 499 Mar 21 } \\ \text { noon }\end{array} & \begin{array}{c}\text { modern } \\ \text { theory }\end{array} & \text { Almagest } & \text { Sunrise } & \text { Midnight } & \text { BSS } \\ \text { Mercury } & 183 ; 25 & 178 ; 01 & 185 ; 24-186 ; 36 & 179 ; 24-180 ; 36 & 180 ; 32-180 ; 50 \\ \text { Venus } & 356 ; 14 & 351 ; 04 & 355 ; 48-357 ; 00 & 355 ; 48-357 ; 00 & 356 ; 48-357 ; 06 \\ \text { Sun } & 359 ; 46 & 357 ; 08 & 0 & 0 & 0 ; 52 \\ \text { Mars } & 6 ; 54 & 4 ; 20 & 6 ; 36-7 ; 48 & 6 ; 36-7 ; 48 & 7 ; 56-8 ; 14 \\ \text { Jupiter } & 186 ; 51 & 185 ; 21 & 186 ; 36-187 ; 48 & 185 ; 24-186 ; 36 & 187 ; 19-187 ; 37 \\ \text { Saturn } & 48 ; 56 & 45 ; 56 & 48 ; 36-49 ; 48 & 48 ; 36-49 ; 48 & 49 ; 00-49 ; 09 \\ \text { Moon } & 280 ; 39 & 278 ; 06 & 280 ; 12-281 ; 24 & 280 ; 12-281 ; 24 & 281 ; 23-281 ; 41 \\ \text { apogee } & 35 ; 26 & 32 ; 24 & 35 ; 06-36 ; 18 & 35 ; 06-36 ; 18 & 37 ; 12-37 ; 30 \\ \text { node } & 352 ; 01 & 349 ; 06 & 351 ; 36-352 ; 48 & 351 ; 36-352 ; 48 & 353 ; 14-353 ; 32\end{array}$

Table 2. Mean longitudes on 499 Mar 21 at noon and $76^{\circ} \mathrm{E}$ from modern theory and ancient theories. As discussed in the text, the values for Mercury and Venus represent the mean longitude on the epicycle, which is to us the heliocentric mean longitude. For the three Indian theories, the only thing known is that the mean longitudes fall into the specified intervals on the date in question. 


$\begin{array}{lcccc}\text { 499 Mar } & \text { Almagest } & \text { Sunrise } & \text { Midnight } & \text { BSS } \\ \text { 21 noon } & & & & \\ \text { Mercury } & -5 ; 25 & (1 ; 59,3 ; 11) & (-4 ; 01,-3 ; 11) & (-2 ; 53,-2 ; 35) \\ \text { Venus } & -5 ; 09 & (-0 ; 26,0 ; 46) & (-0 ; 26,0 ; 46) & (0 ; 35,0 ; 53) \\ \text { Sun } & -2 ; 37 & 0 ; 14 & 0 ; 14 & 1 ; 06 \\ \text { Mars } & -2 ; 34 & (-0 ; 18,0 ; 54) & (-0 ; 18,0 ; 54) & (1 ; 01,1 ; 19) \\ \text { Jupiter } & -1 ; 30 & (-0 ; 15,0 ; 57) & (-1 ; 27,-1 ; 45) & (0 ; 28,0 ; 46) \\ \text { Saturn } & -2 ; 60 & (-0 ; 20,0 ; 52) & (-0 ; 20,0 ; 52) & (-0 ; 05,0 ; 13) \\ \text { Moon } & -2 ; 34 & (-0 ; 27,0 ; 45) & (-0 ; 27,0 ; 45) & (0 ; 43,1 ; 01) \\ \text { apogee } & -3 ; 03 & (-0 ; 20,0 ; 52) & (-0 ; 20,0 ; 52) & (1 ; 46,2 ; 04) \\ \text { node } & -2 ; 55 & (-0 ; 25,0 ; 47) & (-0 ; 25,0 ; 47) & (1 ; 14,1 ; 32)\end{array}$

Table 3. The discrepancies in mean longitude of the four ancient theories with modern theory. The systematic discrepancy for the Almagest results from an overly-long tropical year. 


$\begin{array}{lcccc}\text { 499 Mar } & \text { Almagest } & \text { Sunrise } & \text { Midnight } & \text { BSS } \\ \text { 21 noon } & & & & \\ \text { Mercury } & -2 ; 47 & (1 ; 45,2 ; 57) & (-4 ; 15,-4 ; 57) & (-3 ; 59,-3 ; 41) \\ \text { Venus } & -2 ; 32 & (-0 ; 40,0 ; 32) & (-0 ; 40,0 ; 32) & (-0 ; 32,0 ; 14) \\ \text { Sun } & 0 ; 00 & 0 & 0 & 0 \\ \text { Mars } & 0 ; 03 & (-0 ; 33,0 ; 39) & (-0 ; 33,0 ; 39) & (-0 ; 05,0 ; 13) \\ \text { Jupiter } & 1 ; 07 & (-0 ; 29,0 ; 43) & (-1 ; 41,-1 ; 31) & (-0 ; 38,0 ; 20) \\ \text { Saturn } & 0 ; 23 & (-0 ; 34,0 ; 38) & (-0 ; 34,0 ; 38) & (-1 ; 11,0 ; 53) \\ \text { Moon } & -0 ; 04 & (-0 ; 42,0 ; 30) & (-0 ; 42,0 ; 30) & (-0 ; 23,0 ; 05) \\ \text { apogee } & 0 ; 25 & (-0 ; 35,0 ; 37) & (-0 ; 35,0 ; 37) & (0 ; 40,0 ; 58) \\ \text { node } & -0 ; 18 & (-0 ; 39,0 ; 33) & (-0 ; 39,0 ; 33) & (-0 ; 07,0 ; 25)\end{array}$

Table 4. The discrepancies in mean longitude of the four ancient theories with modern theory after aligning the mean Sun in each theory with the modern theory for the Sun's mean longitude on $499 \mathrm{Mar} 21$ at noon at $76 \mathrm{E}^{\circ}$. 


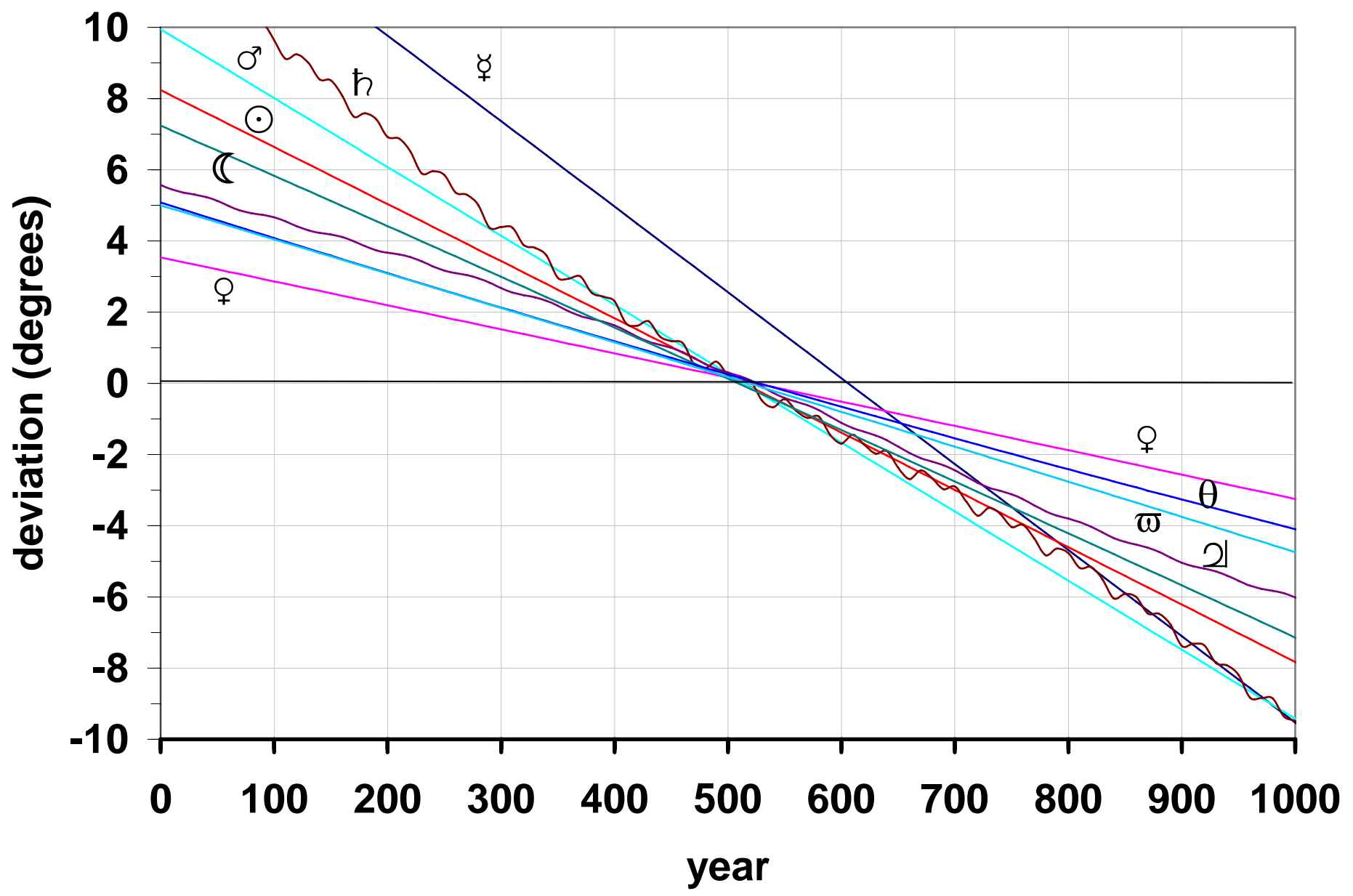

Figure 1. The discrepancies in mean longitude of the sunrise model with modern theory. The single outlier case is Mercury. The lines as shown are as Billard drew them, but are in fact somewhat deceptive, since the sunrise system mean longitudes on any given date are known only to be in an interval of total width $1 ; 12^{\circ}$. The negative slope for each line is due to the fact that the modern mean motions are all less than the sunrise system mean motions. The two lines with small amplitude waves are for Jupiter and Saturn, whose mean longitudes oscillate around a mean value due to the so-called giant resonance. 


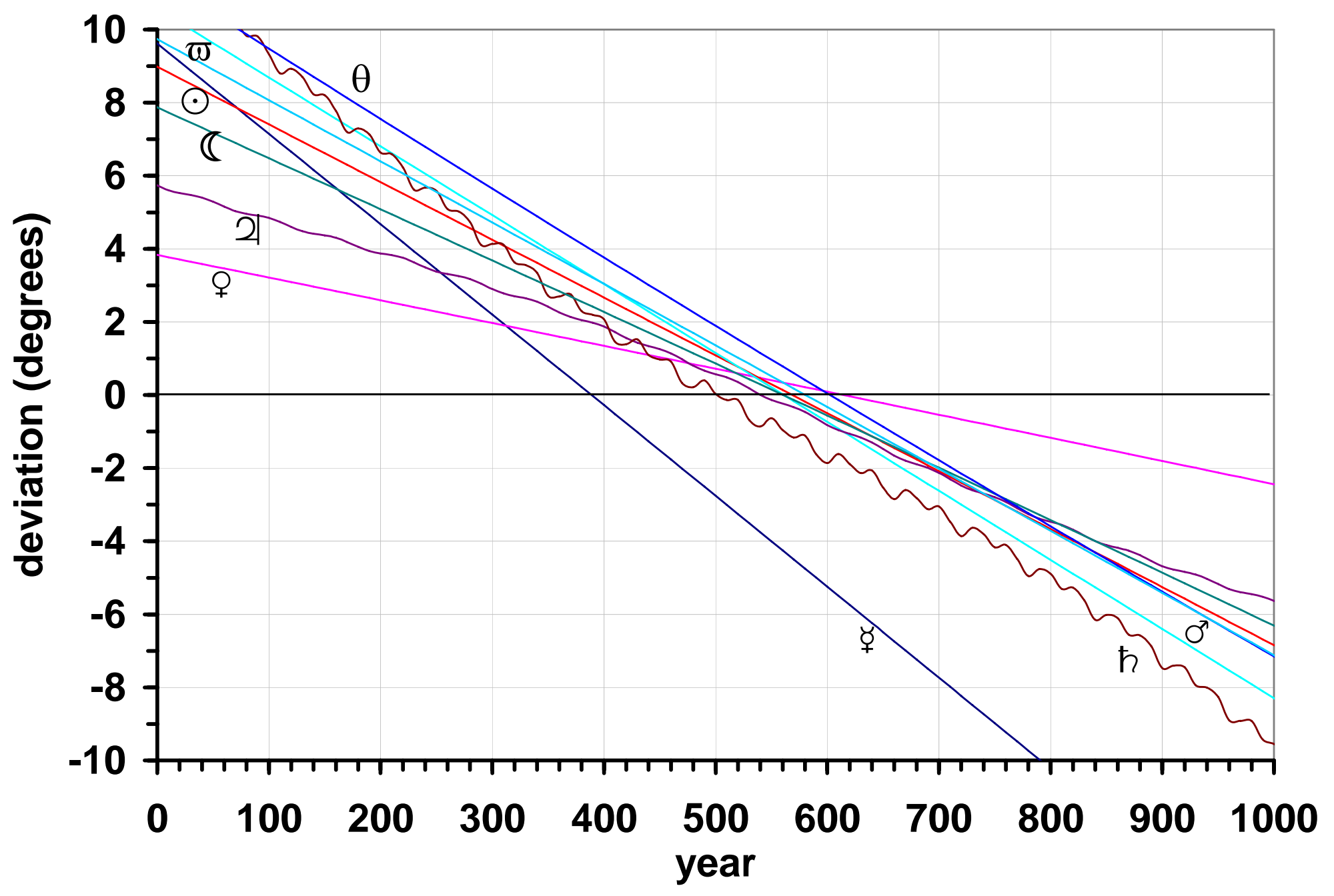

Figure 2. As in Figure 1 except that the lines correspond to the mean longitudes from the Paitamahasiddhanta and the Brahmasphutasiddhanta. The most visible outlier is Mercury, the next most visible is Venus. 


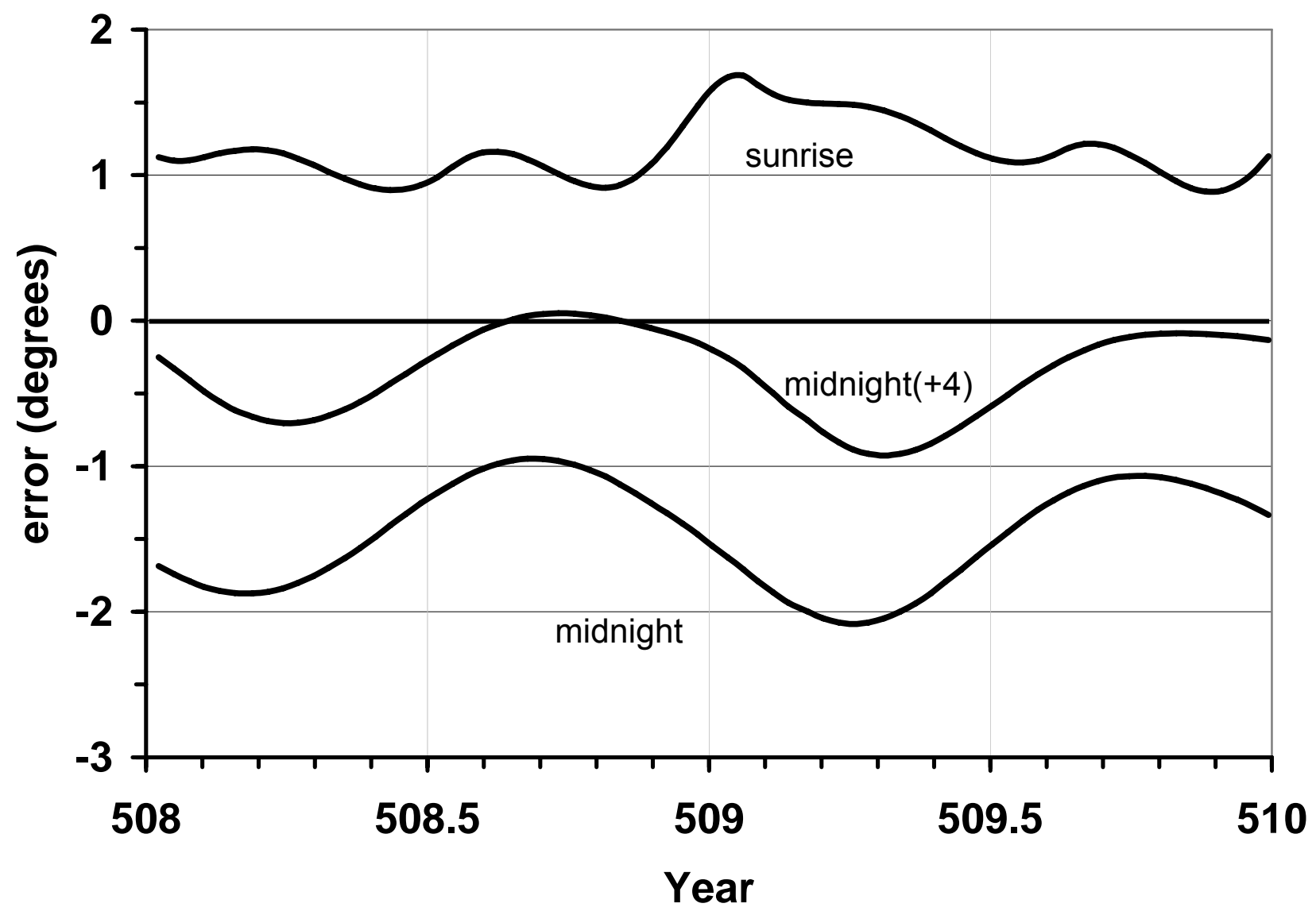

Figure 3. The errors in true longitude for Jupiter over two synodic periods from A.D. 508 -510 . The lower and top curves are for the midnight and sunrise system, while the middle curve is for the midnight system with its revolution number $R$ increased by 4 units, the minimum allowable change. 


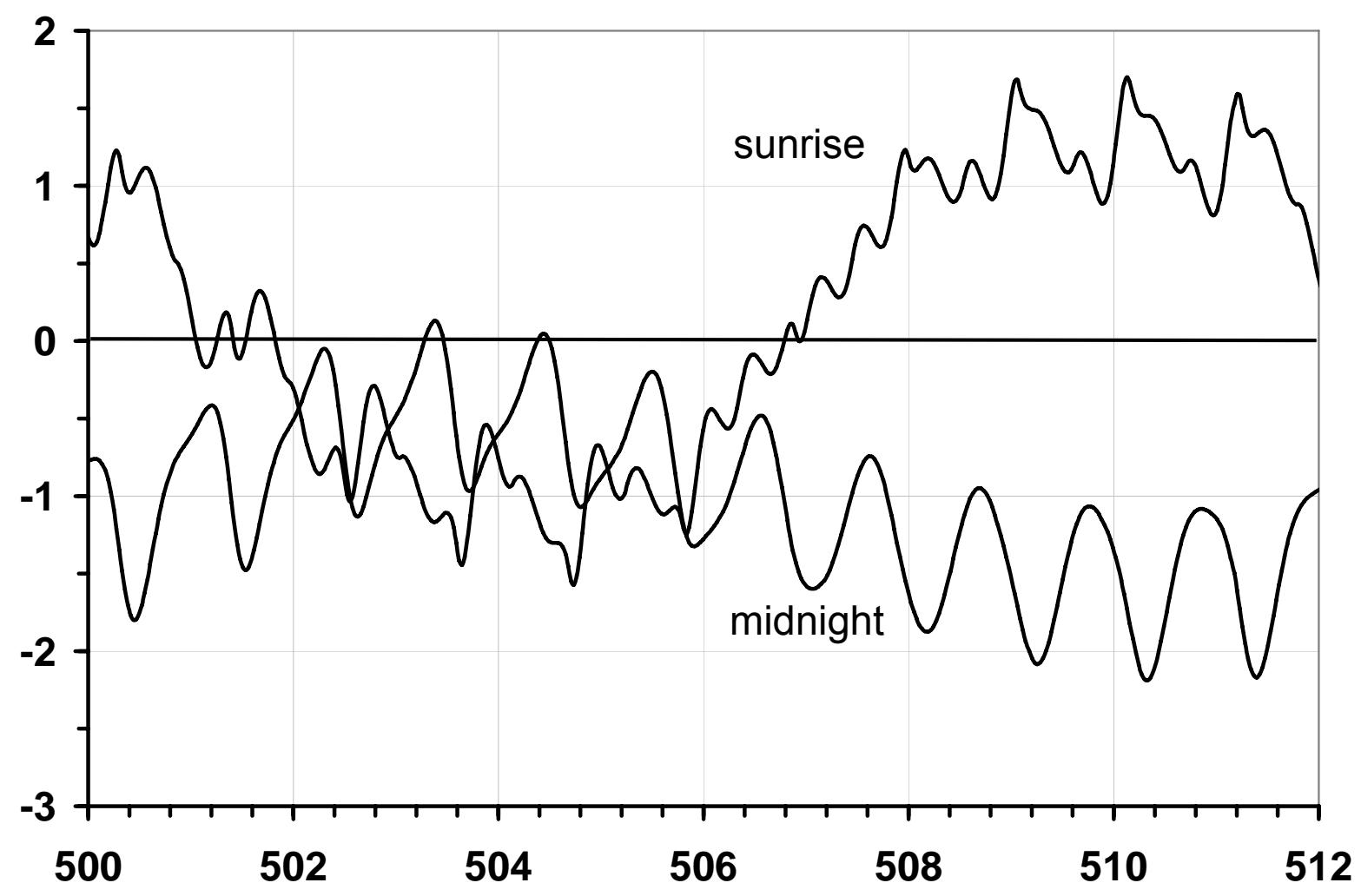

Figure 4. The errors in true longitude for Jupiter for the sunrise and midnight systems over a full longitudinal revolution of 12 years. 


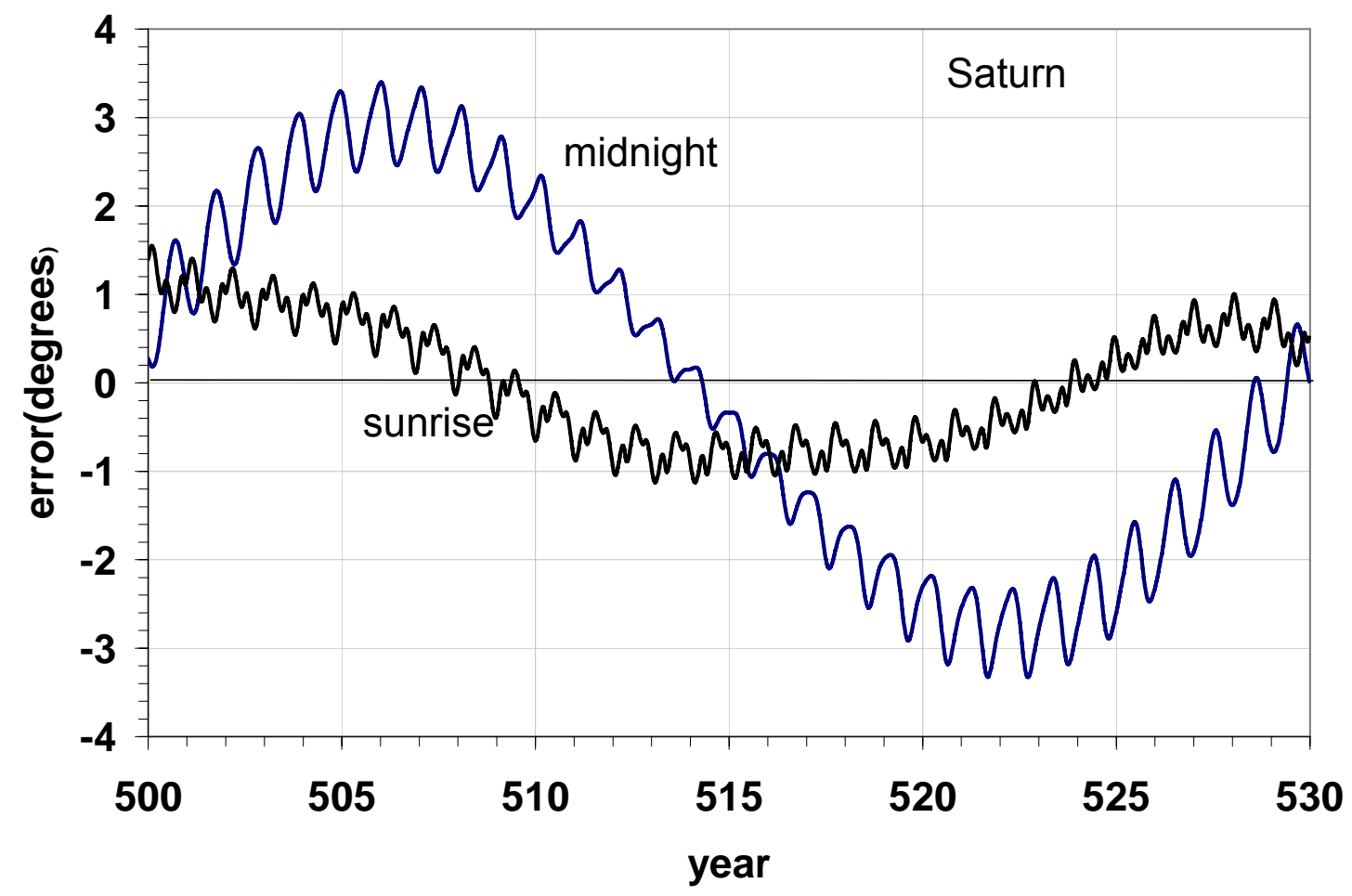

Figure 5. The errors in true longitude for Saturn for the sunrise and midnight systems over a full longitudinal revolution of 30 years. 


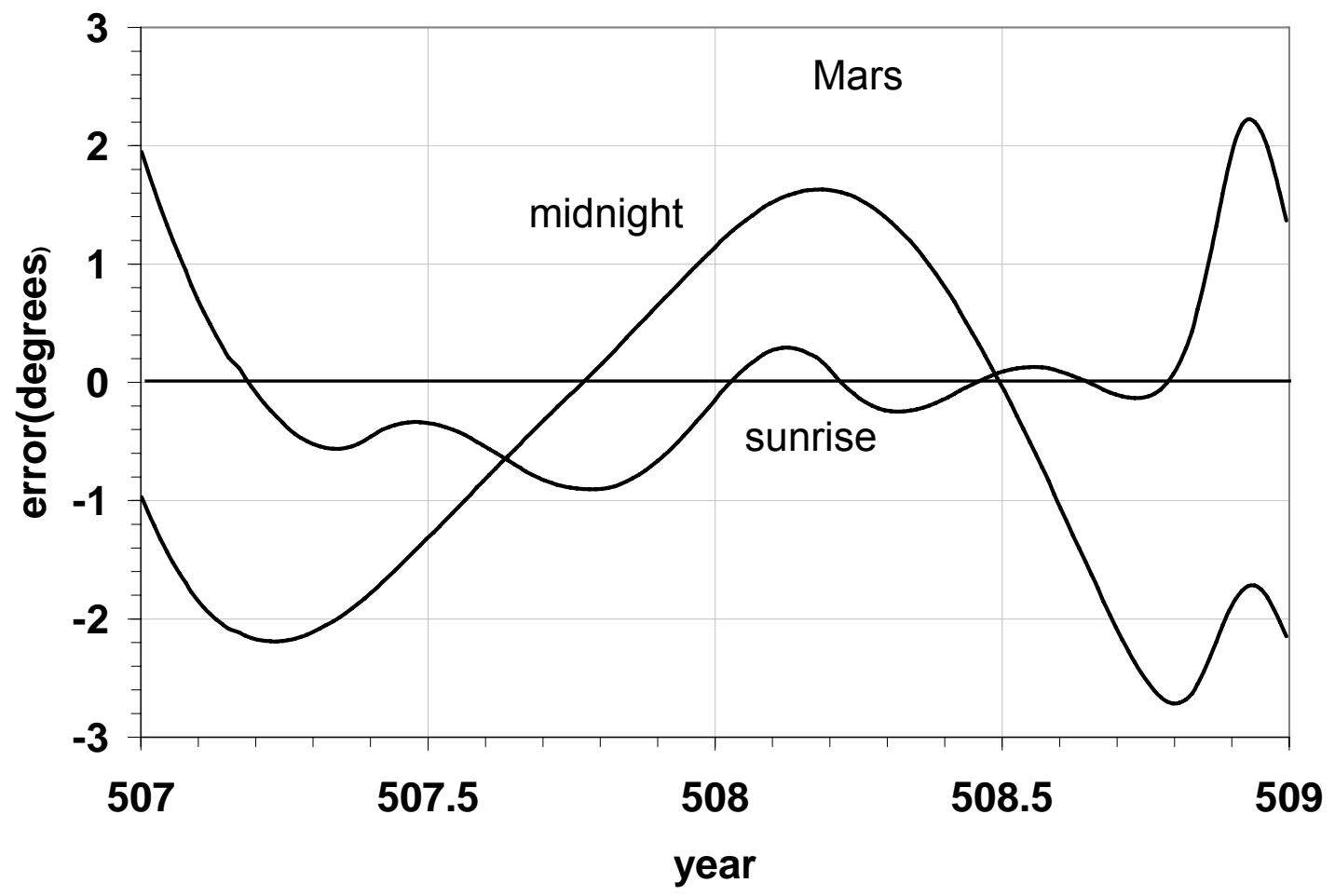

Figure 6. The errors in true longitude for Mars for the sunrise and midnight systems over a full longitudinal revolution of 2 years. 


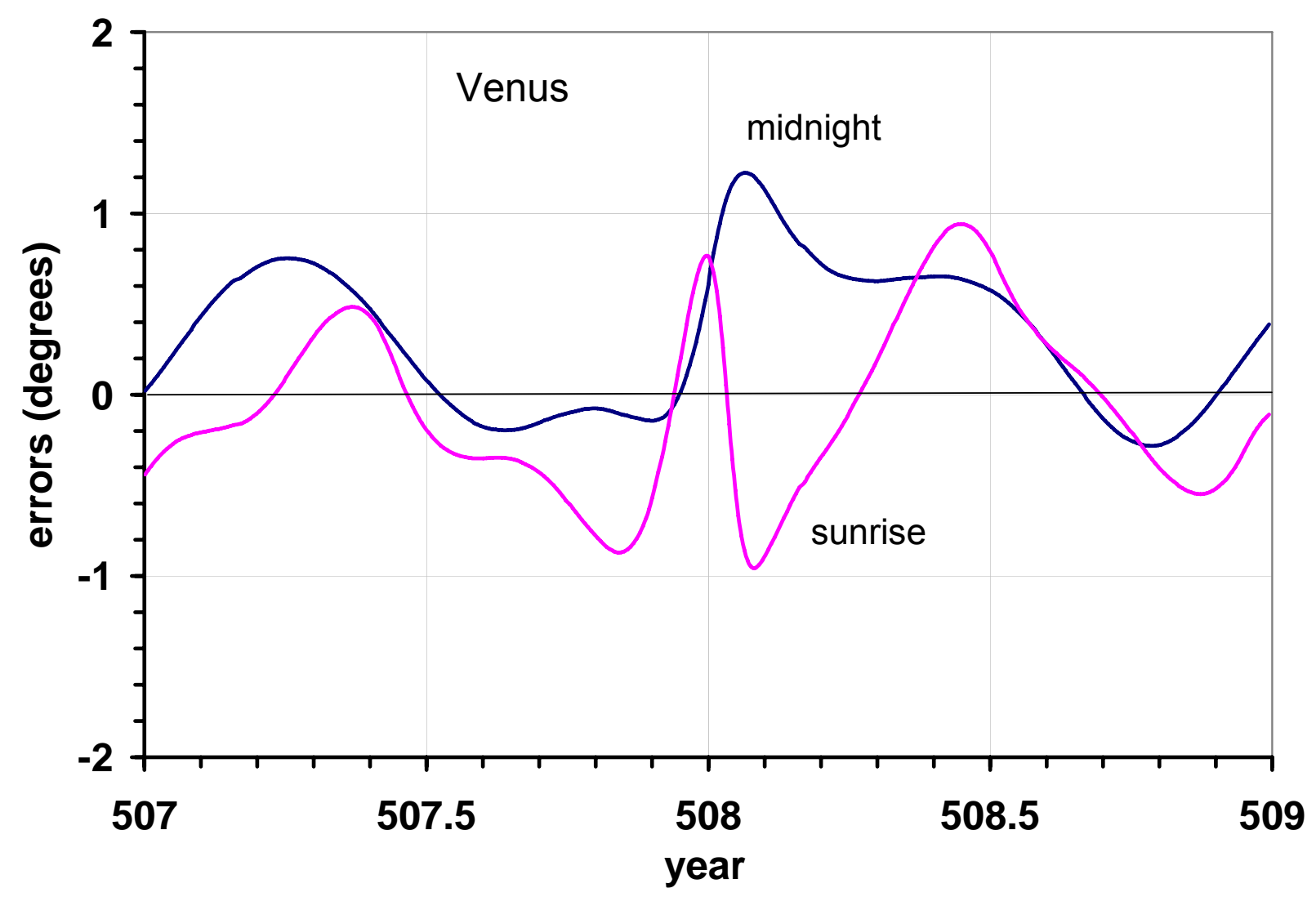

Figure 7. The errors in true longitude for Venus for the sunrise and midnight systems over 2 years. These are longitudes on the epicycle, and so are heliocentric longitudes. 


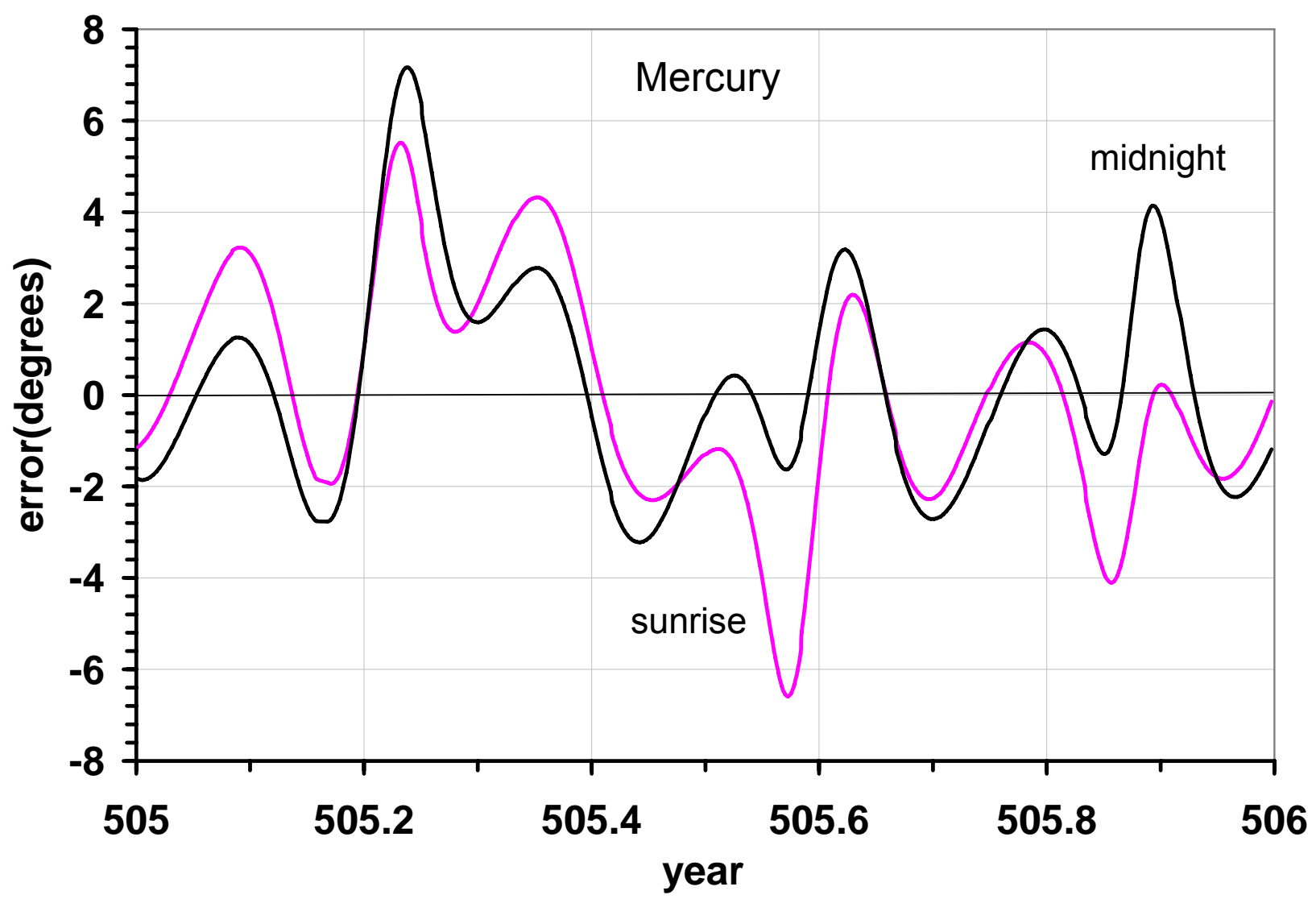

Figure 8. The errors in true longitude for Mercury for the sunrise and midnight systems over 1 year. These are longitudes on the epicycle, and so are heliocentric longitudes. 


\section{REFERENCES}

${ }^{1}$ O. Neugebauer, “The Transmission of planetary theories in ancient and medieval astronomy”, Scripta mathematica, 22 (1956) , 165-192; D. Pingree, “The Recovery of Early Greek astronomy from India”, Journal for the history of astronomy, vii (1976), 109-123; D. Pingree, "History of Mathematical astronomy in India”, Dictionary of Scientific Biography, 15 (1978), 533-633.

${ }^{2}$ G. de Callataÿ, Annus Platonicus: A Study of World Cycles in Greek, Latin and Arabic Sources. Publications de l'Institut orientaliste de Louvain 47. Louvain-la-Neuve, 1996, reviews all the relevant source material. A. Jones, "The Keskintos Astronomical Inscription: Text and Interpretations”, SCIAMVS, 7 (2006), 3-42, is the definitive analysis of the Keskintos inscription, the clearest use of a great year in a Greco-Roman source; B. L. van der Waerden, “The Great Year in Greek, Persian and Hindu Astronomy”, Archive for history of exact sciences, 18 (1978), 359-383 is a good presentation of the evidence, but his conclusions are not solidly proven.

${ }^{3}$ G. J. Toomer, Ptolemy's Almagest (1984), 423-6.

${ }^{4}$ O. Neugebauer, A history of ancient mathematical astronomy, (1975), 380-468.

${ }^{5}$ Toomer, ibid. (ref. 3), Books IV-V for the Moon and Books IX-XI for the planets. ${ }^{6}$ O. Neugebauer, ibid. (ref. 4), 905-908.

${ }^{7}$ K. S. Shukla, Aryabhatiya of Aryabhata (1976) is the sunrise system; K. S. Shukla, Mahabhaskariya of Bhaskara I (1960) is a commentary on the sunrise system; O. Neugebauer and D. Pingree, The Pancasiddhantika of Varahamihira (2 vols, Copenhagen, 1970-71) contains the midnight system; $\mathrm{B}$. Chatterjee, The Khandakhadyaka of Brahmagupta (1972) is a commentary on the midnight system; D. Pingree, "The Paitamahasiddhanta of the Visnudharmottapurana", Brahmavidya, xxxixxxii (1967-68), 472-510.

${ }^{8}$ S. Prakash, A Critical Study of Brahmagupta and His Works. (Dehli, 1986), 289-314.

${ }^{9}$ L. González-Reimann, Tiempo Cíclico y eras del Mundo en la India, (Mexico, 1988) and L. González-Reimann, The Mahabharata and the Yugas: India's Great epic Poem and the Hindu System of World Ages, (NewYork, 2002).

${ }^{10}$ The Mahabharata, translated by Kisari Mohan Ganguli, (1883-1896), Book 12: Santi Parva, Section 231 (see http://www.sacred-texts.com/hin/m12/m12b058.htm).

${ }^{11}$ H. H. Wilson, The Vishnu Purana, (London, 1840), Book 1 Chapter 3, Book 3 Chapters 1-3, Book 4 Chapter 1, and Book 5 Chapter 23 (see http://www.sacredtexts.com/hin/vp/index.htm). ${ }^{12}$ P. Olivelle, The Law Code of Manu, (Oxford, 2004), 1.61-86. An older version, G. Bühler, The Laws of Manu, Sacred Books of the East, vol. 25 (Oxford,1886), is available (at least in some countries) at http://books.google.com. See in particular pp. lxxxii ff. ${ }^{13}$ D. Pingree, The Thousands of Abū Mashar, (London, 1968), 28-29.

${ }^{14}$ D. Pingree, ibid. (ref. 1, 1976) 117-120, and H. Thurston, "Medieval Indians and the Planets", DIO 8.1 (1998) 18-20, give incomplete accounts of the following procedure. Pingree in particular claims that the construction of the $R$ values uses only period relations but no mean longitudes at the modern date. 
${ }^{15}$ Incidentally, every one of the 10,000 four digit integers between 0 and 9999 is the final four digits of one and only one multiple of the form $n \times 1097$, with $n$ ranging over 0 to 9999.

${ }^{16}$ D. Pingree, ibid (ref. 1); D. Pingree, "Aryabhata, the Paitamahasiddhanta, and Greek Astronomy”, Studies in History of Medicine \& Science, XII 1-2 New Series (1993), 6979.

${ }^{17}$ R. Billard, L'astronomie Indienne, (Paris, 1971); R. Billard, “Aryabhata and Indian Astronomy”, Indian Journal of History of Sciences 12 (1977) 207.

${ }^{18}$ B. L. van der Waerden, “Two Treatises on Indian Astronomy”, Journal for the history of astronomy, xi (1980), 50-62.

${ }^{19} \mathrm{D}$. W. Duke, "The equant in India: the mathematical basis of Indian planetary models", Archive for History of Exact Sciences, 59 (2005) 563-576.

${ }^{20} \mathrm{~B}$. L. van der Waerden, ibid. (ref. 15) uses this example to argue, incorrectly as we shall see, that it would have been straightforward for Aryabhata to use a few observations to change the midnight scheme parameters into the sunrise scheme parameters.

${ }^{21}$ R. Mercier, "The Standard Scheme of the Moon and Its Mean Quantities", Archive for History of Exact Sciences, 61 (2007) 255-272. 